\title{
The geometry of craniofacial growth invariants
}

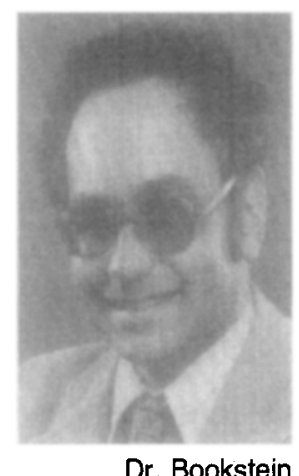

\begin{abstract}
Fred L. Bookstein
Ann Arbor, Mich.

In the description of craniofacial growth it is crucial to identify constant geometric relations. The search leads to complex manipulations of coordinate data from cephalograms. In the regularities that he finds, the clinician must be aware of the difference between empirical contingency and geometric necessity. For instance, common procedures for identifying centers away from which growth proceeds radially (the "polar phenomenon") ignore theorems about the indeterminacy of such centers. The scan for growth invariants in configurations of landmarks should be based on the method of tensor analysis. Any shape change may be summarized in terms of two directions in each triangle of landmarks. The directions are at 90 degrees in each triangle both before growth and after; one of them bears the greatest percent rate of change of length of all directions in the triangle, and the other bears the least. From a diagram of these axes one comes immediately to strict invariants of the shape change-specific angles and proportions along segments defined by relations between the triangle and the axes-linked to other specific angles and proportions which change most rapidly. It is simple to adapt this machinery so that it can deal with average shape changes in populations. Included here are two examples of the technique: (1) extracting the statistically most stable definition of "growth axis" (displacement of menton from cranial base) in children aged 6 to 14 years and (2) describing the shape change of the rat calvarium between the ages of 7 days and 150 days. Such descriptions should be the first step in designing any cephalometric analysis.
\end{abstract}

Key words: Cephalometrics, craniofacial growth, shape change, biorthogonal analysis, Cartesian transformations

$\mathrm{O}_{\mathrm{r}}$ rthodontists familiar with the extraction of cephalometric "'analyses"' by tangible ruler and protractor are not prepared for critical thinking about more advanced analyses - construction of superpositions and configurations, growth "prediction" by manipulation of vectors, facial typing. In spite of the growing availability of digitizing tablets, there has been no parallel distribution of conceptual tools for making sense of all these Cartesian coordinate data. One is at a loss to distinguish in the literature between phenomena contingent upon the data ("findings") and geometric necessities. This article urges an enlightened exploitation of the geometric necessities to produce carefully regulated findings.

The difference between theorems and findings is especially crucial for descriptions of growth, the literature of which supplies us with considerably more conjectures than refutations. The main biometric component of growth analysis is the detection of invariants, relations which (unless interfered with) appear not to change their average values over time. For example, to "predict" craniofacial form is to extrapolate the

From the Center for Human Growth and the Department of Radiology, The University of Michigan. geometric consequences of some invariance under increase in size.

This essay describes some geometric manipulations by which mean growth invariants can always be extracted from observed configurations of landmarks. Reports of constancies empirically observed, such as "polar phenomena" or "growth axes," need to be judged against these automatic report-gencrating mechanisms in order that the serious investigator may know what is real and what is artifact. That craniofacial shape change after infancy is rather small engenders many paradoxes, inefficiencies, and fallacies of measurement. The ubiquity of invariants allows us to put some fairly stringent requirements on cephalometric quantification without losing the information borne in our data.

\section{THE NOTION OF "GROWTH CENTER": A CRITIQUE}

In recent years several authors ${ }^{1-3}$ have independently put forward a general class of growth models that share the same fundamental fallacy.* Here I dissect

*The practice of this fallacy is not limited to orthodontics. It also appears in cardiology, for instance, in the course of attempts to describe cardiac contraction using polar coordinates. 
(a)

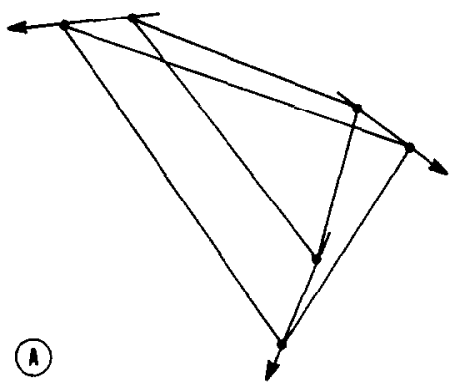

(B)

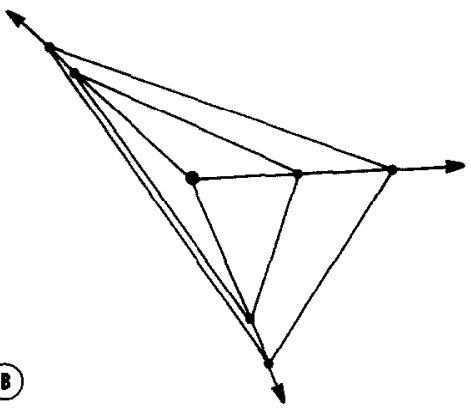

(c)
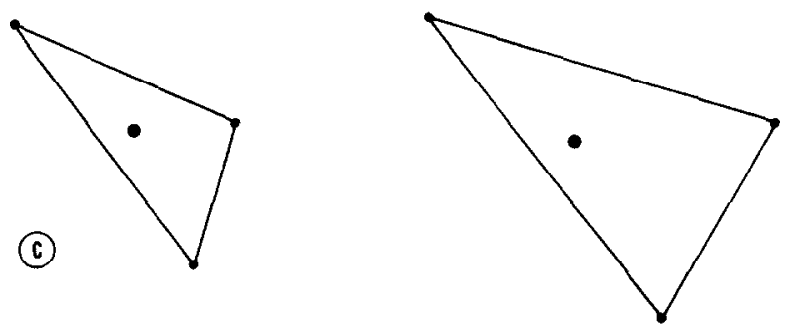

Fig. 1. Most superpositions (A) of two triangles $P_{1} P_{2} P_{3}, Q_{1} Q_{2} Q_{3}$ do not suggest a growth center. However, if the lines joining corresponding landmarks meet in a point (B), that point takes on the status of a double landmark $P_{C}=Q_{C}$, one site in each triangle $(C)$, with respect to which growth appears to be pure radial dilation.

this notion as a caution against proceeding from coordinates to "measurement" without a thorough geometric exploration. The exposition here is condensed from another study of mine. ${ }^{4} \mathrm{~A}$ refutation of a quite different sort may be found in a forthcoming work by Moss and associates. ${ }^{5}$

\section{Triangles}

Consider a set of three landmarks in each of a pair of cephalograms. For illustration, use $P_{1} P_{2} P_{3}$ for the vertices of the first triangle and $\mathrm{Q}_{1} \mathrm{Q}_{2} \mathrm{Q}_{3}$ for the corresponding vertices of the second. Suppose these two triangles are drawn on clear plastic sheets freely superimposable as to both translation and rotation. For any superposition, we may draw the lines connecting the two images of each landmark-the lines $P_{1} Q_{1}$, $\mathrm{P}_{2} \mathrm{Q}_{2}, \mathrm{P}_{3} \mathrm{Q}_{3}$. For most superpositions the lines are not

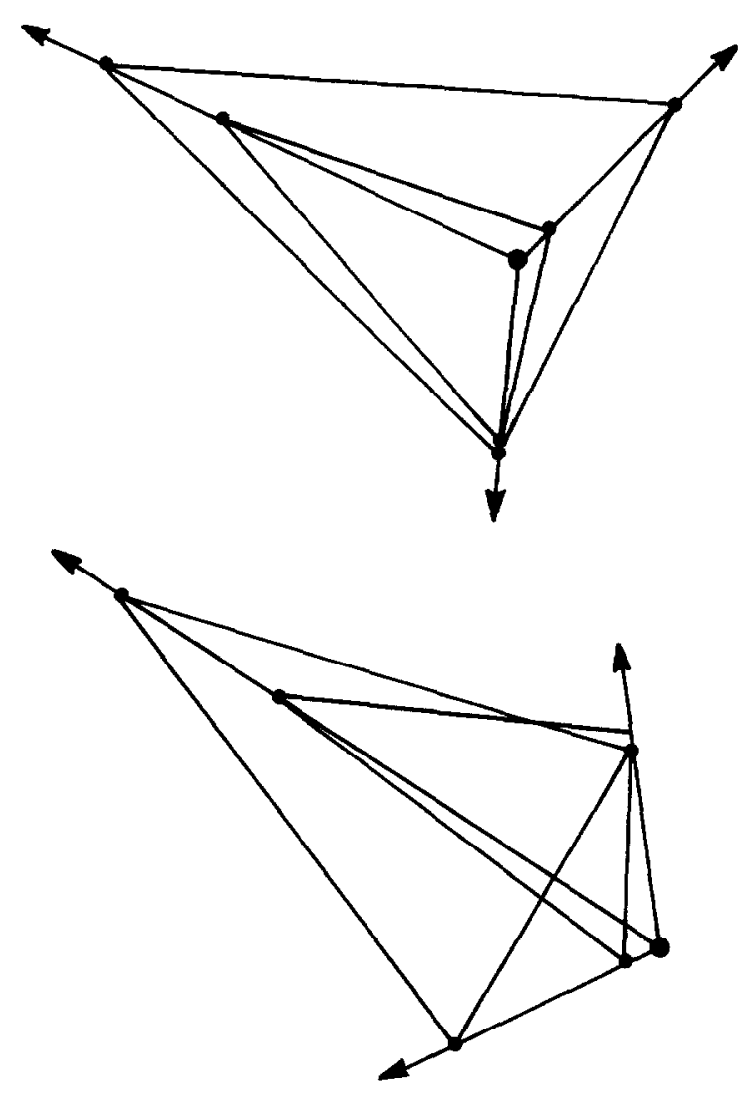

Fig. 2. For each of two other arbitrary points in the plane of the triangle $P_{1} P_{2} P_{3}$, a superposition in which it serves as growth center. These were constructed by the routine described in the Appendix.

helpful (Fig. 1, $A$ ). However, should they meet in a point (Fig. 1, $B$ ), they suddenly become strongly suggestive of a model for growth. We are tempted to interpret the point of concurrence of the lines as a pair of points $\mathrm{P}_{c}, \mathrm{Q}_{\mathrm{c}}$, one in the plane of each triangle (Fig. $1, C)$. From this point, which now has the import of a newly constructed landmark, growth appears to be pure radial dilation. The P's seem to grow directly away from the center, at various rates, until by the second cephalogram they just overlie the Q's. In particular, the angles $\angle \mathrm{P}_{1} \mathrm{P}_{\mathrm{c}} \mathrm{P}_{2}=\angle \mathrm{Q}_{1} \mathrm{Q}_{\mathrm{c}} \mathrm{Q}_{2}$, etc. are apparent invariants of the growth process. The enthusiastic observer is tempted to weave this single diagram into a whole fabric of predictions and analyses. Let me refer to these special superpositions, so suggestive, as isogonal superpositions and to the points $\mathrm{P}_{\mathrm{c}}$ and $\mathrm{Q}_{\mathrm{c}}$ as a pair of isogonal centers.

Alas, it is a most unfortunate geometric fact (see Appendix) that any point whatever of either form may serve as an isogonal center in this sense (Fig. 2). Therefore, such a superposition tells one nothing at all about growth; the construction is, instead, a tautology of 

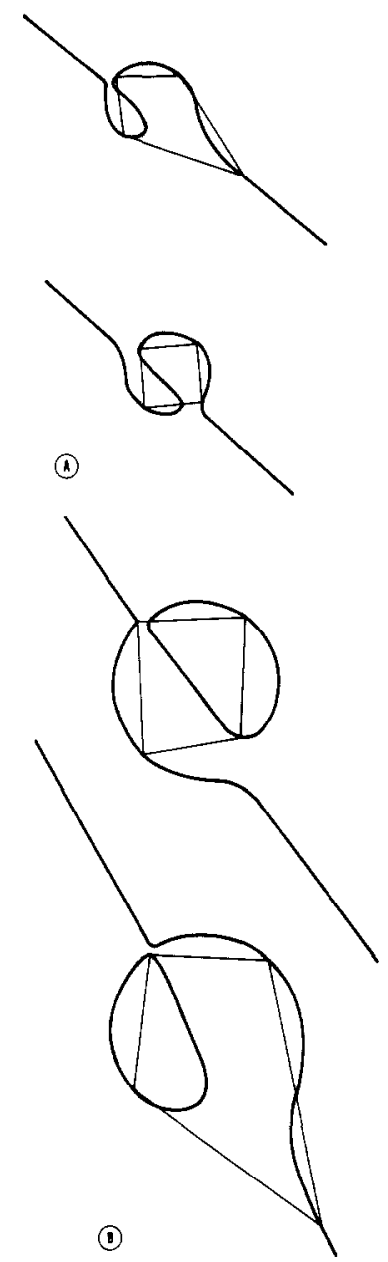

Fig. 3. Two examples of the curve of isogonal centers for a pair of quadrilaterals.

Euclidean geometry, and the pairing of points which emerges has no biologic meaning.

\section{Quadrilaterals}

Since this result is unpleasant to contemplate, let us demand a bit more of the "centers" we are trying to find: lct us require that they be isogonal with respect to four points. If the $\mathrm{P}$ polygon is exactly similar to the $\mathrm{Q}$ polygon, then we can again superpose at any point in the first polygon. Otherwise, to the extent that size change bears a gradient across the form, angles measured to a central landmark will not change as much as angles measured to a landmark on the periphery, so that any anatomic superposition near the center of the form will appear to work fairly well. The main theorem deals with such configurations, whatever the landmarks and wherever their locations:

For any two landmark quadrilaterals $\mathrm{P}_{1} \mathrm{P}_{2} \mathrm{P}_{3} \mathrm{P}_{4}$, $\mathrm{Q}_{1} \mathrm{Q}_{2} \mathrm{Q}_{3} \mathrm{Q}_{4}$ that are not exactly similar, the col- lection of possible isogonal centers $P_{c}$ in the $P$ plane comprises one or two whole continua of points, a complete curve of isogonal superpositions. To each point $\mathrm{P}_{\mathrm{c}}$ of this curve corresponds a unique point $Q_{c}$ on a similar curve in the Q plane.

The curves to which the theorem refers look like the specimens shown in Fig. 3. The nineteenth-century geometer knew them under the name of circular cubics. Each has the underlying structure of the Greek letter $\phi$, a circle cut by a line, about which a curve is then threaded in one circuit or two. Each curve passes through all four landmarks of its plane and has one single asymptote as it tends to infinity in two opposite directions; each curve is a function of both quadrilaterals of points. Fig. $4, A$ shows a portion of such a pair of curves and the pairing of centers along them; Fig. 4, $B$ and $C$ shows typical exact "polar" superpositions based upon the pairing.

From this continuum of possibilities, we can select specific point-pairs only if we revert to biologic criteria. A growth center that is biologically meaningful-a fifth, "fixed" landmark - must be biologically homologous from form to form. However, the curves which $P_{c}$ and $Q_{c}$ traverse have been computed without any regard for biologic homology. In general, they are not homologous for any pair of corresponding centers. It follows that, for most pairs of quadrilaterals, no such superposition makes any particular sense.

When growth is slow, as with the craniofacial skeleton, the two quadrilaterals being compared are nearly similar. Then the arcs of the isogonal curves are themselves somewhat homologously placed, so that there will be ostensible approximate centers at the point of the curves where $P_{c}$ and $Q_{c}$ are most nearly homologous. Inspection of this curve is necessary for construction of meaningful growth centers from empirical data. For instance, consider the two forms in Fig. 5 , adapted from Fig. 12 of Ricketts, ${ }^{1}$ and the growth center PT that Ricketts identifies. Segments condylion-menton, menton-ANS, ANS-nasion, and nasioncondylion of the smaller form in Fig. 5 span angles of $119,45.5,42.5$, and 153 degrees at PT, and corresponding segments of the larger form span angles of $121.5,40.5,44.5$, and 153.5 degrees, but the true locus of isogonal centers (Fig. 6, $A$ ) passes nowhere nearby. The situation is the same when we replace menton with PNS (Fig. 6, B). The fact that angles are approximately unchanged at PT does not imply that PT is at all an approximation of a proper isogonal center.

For configurations of five landmarks, one's choice of exact centers is restricted to pairs of isolated points 


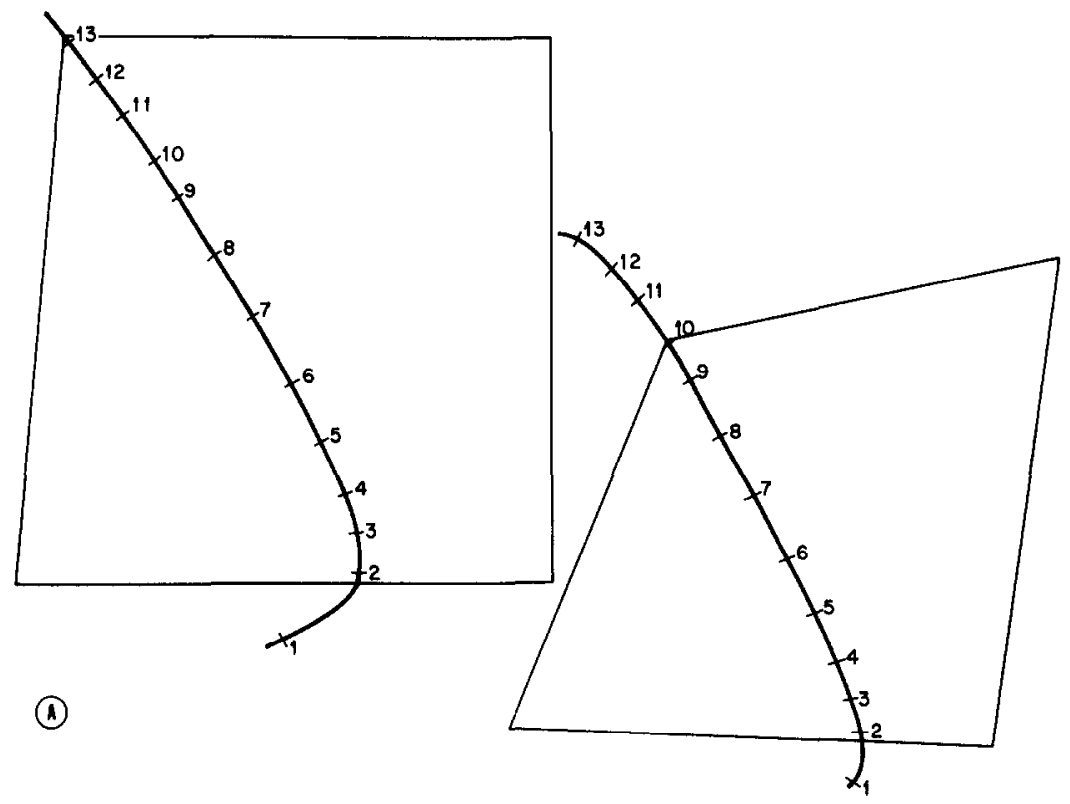

(B)
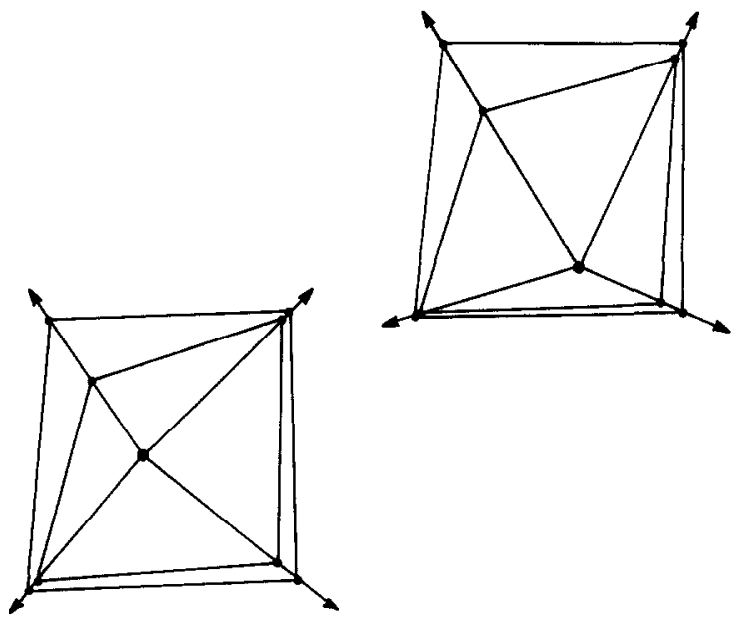

Fig. 4. A, A portion of such a pair of curves, with the isogonal pairing explicitly indicated. B, Two exact isogonal superpositions of the two quadrilaterals on centers selected from the curves in $\mathbf{A}$.

whose biologic homology between images is highly unlikely. For more than five landmarks, exact centers cannot be expected to exist. Computations can proceed only by least-squares methods ${ }^{5}$ that lead to uselessly broad confidence regions.

This notion of the growth center has, in my view, no biometric import. It is mainly an entanglement of the slowness and near-similarity of craniofacial growth with improper intuitions about geometry. Yet we know intuitively that in the growing head some angles or proportions grow and others diminish, so that some must be invariant. How may we find them? And how might they aid in simple summaries of shape change?

\section{FOUNDATIONS OF SHAPE COMPARISON}

Morphometrics is the interplay between geometric location and biologic homology. ${ }^{6}$ Its successful analyses are all variants of a single approach, the modeling of shape change as deformation. This section briefly explains the fundamental method for measuring these changes. This article is one of a series applying the method to various cephalometric problems. The application to treatment effects is dealt with in two other studies $^{7,8}$ which present the basic strategies rather less tersely. The application to population mean differences is indicated in an article by Moyers and Bookstein ${ }^{9}$ and to diagnosis of anomalies in a work now in preparation by Grayson and colleagues. ${ }^{10}$ 


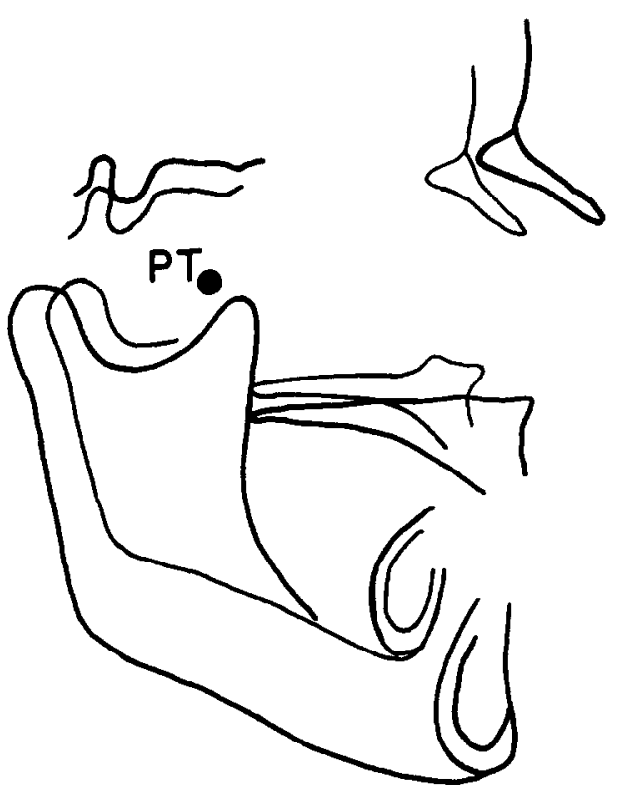

Fig. 5. Ricketts"1 "composite at age 8 and 18," including the point PT he suggests as the center of the "polar phenomenon." (Adapted from McNamara, J. A., Jr., editor: Determinants of Mandibular Form and Growth, Ann Arbor, 1975, Center for Human Growth and Development, University of Michigan.)

The measurement of biologic shape change is quite different from the measurement of shape. It is not managed by measuring shapes separately and then subtracting scores on corresponding measures. Rather, shape change is a geometric object in its own right, the deformation of one form into another which accords with biologic homology. Of the information relevant to the analysis of shape change, that which is biologic is manifested in the homology map, a function which assigns a correspondence between the points of any pair of forms. This homology map is a mathematical model of biologic homology as usually construed, which consists of spatial or ontogenetic correspondences between structures or parts. ${ }^{6,}{ }^{11}$ We generally sample this function at landmarks, points reliably located by anatomic criteria. (That landmarks are homologous is taken for granted in the discussion here.) The coordinate system of any form separately is inessential. It is the relation between two coordinate systems for homologous configurations of points that drive the computation of shape change.

For sufficiently small regions, shape changes may be modeled as uniform. They are described by the rate of change of length, or dilatation, as a function of direction. Dilatation is measured as a ratio, not a difference, of homologous lengths. It is dimensionless. The essence of the biorthogonal method is the summary of the dilatations by the largest and smallest at any point. These are called the principal dilatations. The direc-

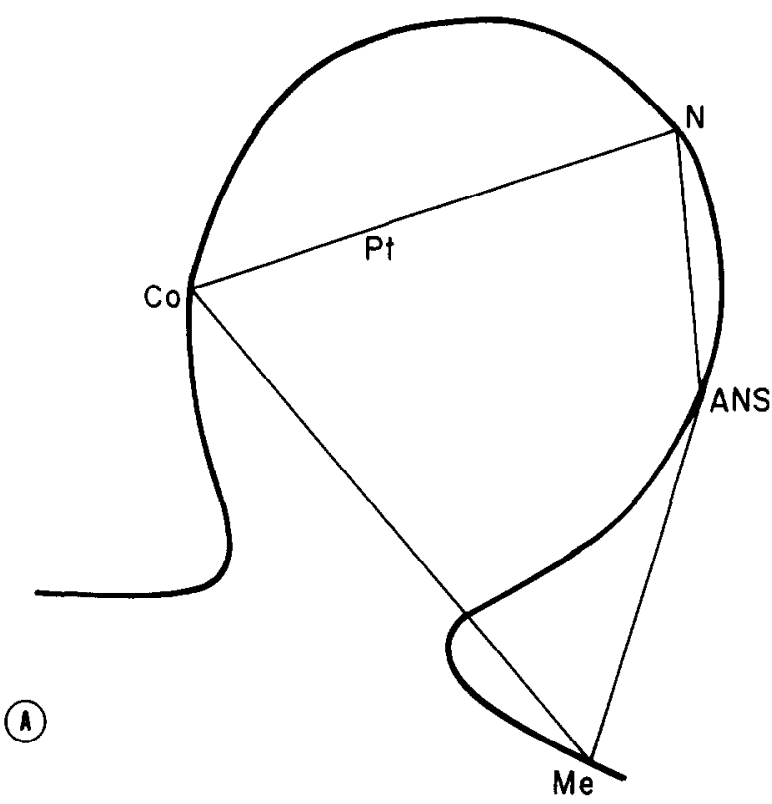

(B)

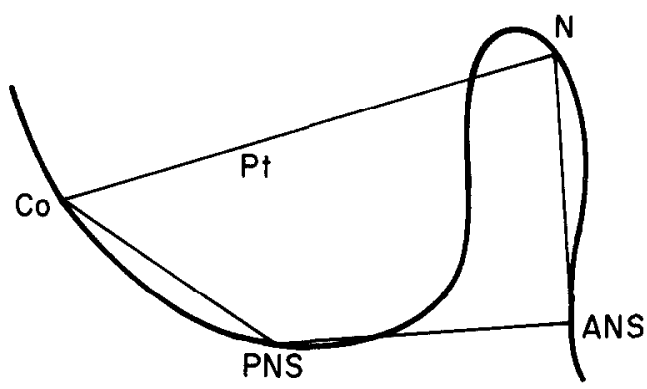

Fig. 6. Exact curves of isogonal centers for the 8-year-old configuration shown in Fig. 5. A, For the quadrilateral condylion-menton-ANS-nasion. B, For the quadrilateral condylionmenton-ANS-PNS.

tions along which they lie, the principal axes or biorthogonal directions, are at exactly 90 degrees both before and after transformation.

For expository purposes, we may take the basic unit of analysis to be a homologous pair of triangles of landmarks, as in Fig. 7.* In the absence of other information, we may take the transformation sampled by these limited data to be uniform between homologous pairs of edges and throughout the interiors of the triangles. The homogeneity of the transformation is indicated

*These triangles are not to be thought of as biologically real. Their sides may pass through various tissues or through air. They merely provide a convenient graphic formalism for describing changes in the configuration of their vertices, the landmarks whose locations are the real biometric data of these examples. Of course, the method is not limited to landmarks in sets of three. Extended polygons can be studied by the use of alternate triangulations, as in the second example of this essay, or by a tensor field that is not uniform, as represented in the curving biorthogonal grid. ${ }^{11}$ 


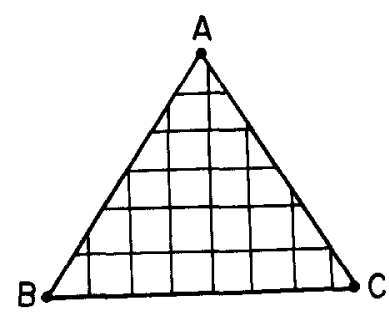

(a)
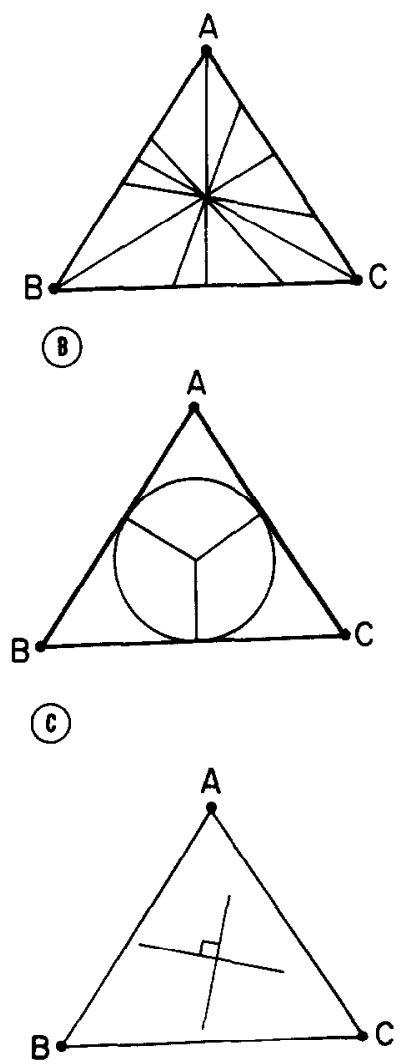

(B)

(C)

(D)
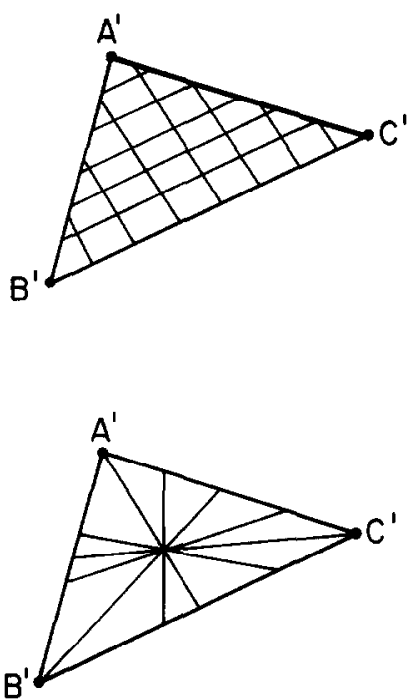
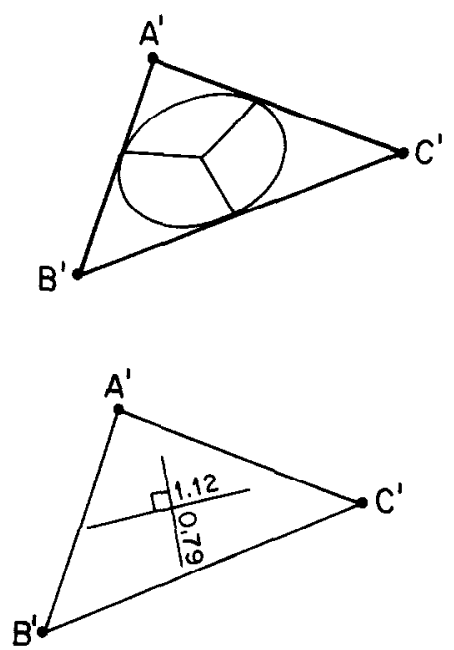

Fig. 7. The method of biorthogonal directions for two triangles. A, The uniform transformation of interiors they suggest. B, Dilatations of lengths in various directions. C, Dilatations may be represented by the radii of the ellipse into which a circle is deformed. D, The principal directions are axes of this ellipse, and the principal dilatations are proportional to their lengths.

clearly in the transformation grid after the style of D'Arcy Thompson (Fig. 7, $A$ ).

We may draw the transformation just as clearly in terms of the collection of lines in all directions (Fig. $7, B)$. The deformation we are observing, driven by the displacements of those landmarks at the corners, will deform these lines into others which divide the edges in the same fractions. That is, the deformation takes edges to edges, median lines (dividing the opposite sides in the ratio of 50:50) to medians, and so on.

\section{Principal axes computed from triangles of landmarks}

We are interested in the ratios of lengths of corresponding lines in the two triangles, the dilatations. We could compute them by taking quotients of corresponding lengths, direction by direction, but instead we may observe the dilatations explicitly as the lengths of the deformations of lines of constant length, that is, of radii of a circle. We can actually draw a circle (Fig. 7,C) whose deformation we wish to observe and the oval into which the uniform shear takes it. The dilatations of 


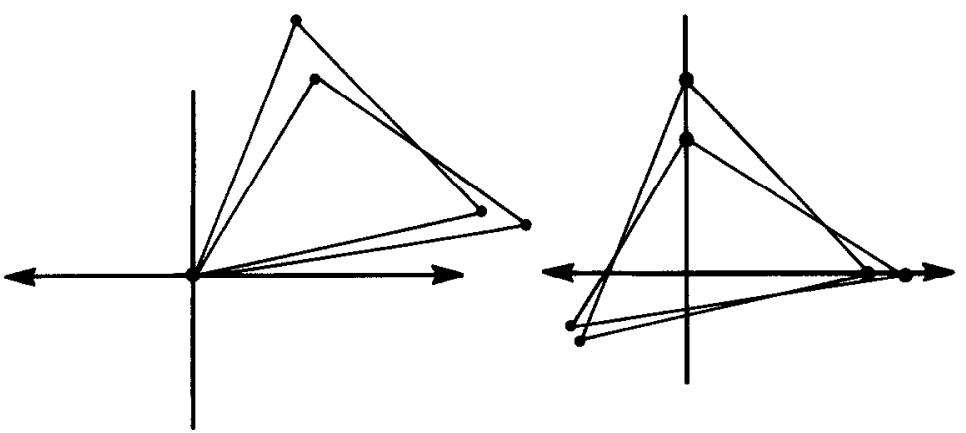

Fig. 8. Two of the indefinitely many superpositions, equally valid, suggested by the analysis in Fig. 7 .
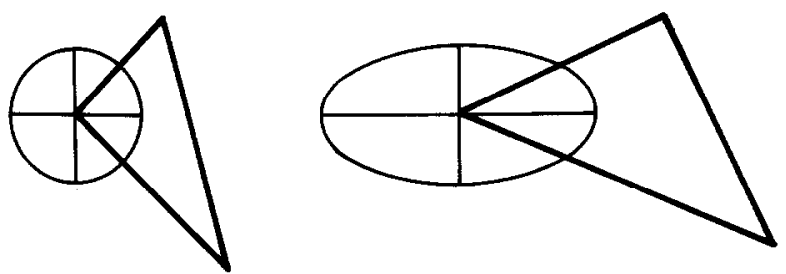

Fig. 9. Invariant ratios. Segments at equal angles to the princi$\mathrm{pal}$ axes of a deformation are dilatated at the same rate.

line-elements are proportional to the radii of this oval.

An accurate drawing shows that this oval is remarkably like an ellipse. In fact, it is an ellipse, exactly. The image of the circle under growth has two axes of symmetry, which lie at 90 degrees. One is the largest diameter of the ellipse, one the smallest. The diameters of the circle which transform into them are likewise at 90 degrees.

It should be recalled that the lengths of the radii embody the dilatations as a function of direction. Then the principal axes of the ellipse into which a circle is taken are the principal directions of the deformation as they lie upon the right-hand form. The diameters that were mapped into them are determined by corresponding fractions of intersection along edges of the triangles. In Fig. 7, $D$ they have been drawn without their ovals. The dilatations indicated on the figure were computed by division of lengths of homologous segments; dilatations in any other direction may be computed from these two according to the formula

$$
\mathrm{d}^{2}(\theta)=\mathrm{d}_{1}^{2} \cos ^{2} \theta+\mathrm{d}_{2}^{2} \sin ^{2} \theta
$$

where $\theta$ is the angle (in the left-hand form) between the direction and the axis of dilatation $d_{1}$.

The analysis separates the observed change into one component for size change and a second component for shape change. The product of the dilatations is the ratio by which the area of the triangle has increased; their quotient is a measure of the anisotropy, or directionality, of this size change. One may think of any distortion
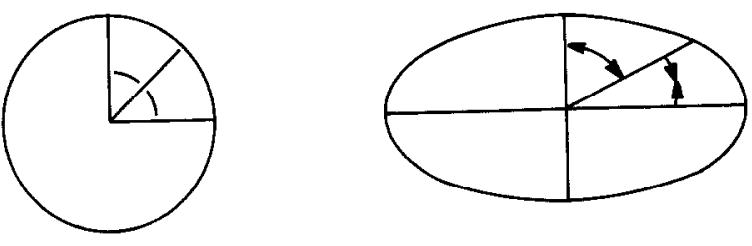

(1)
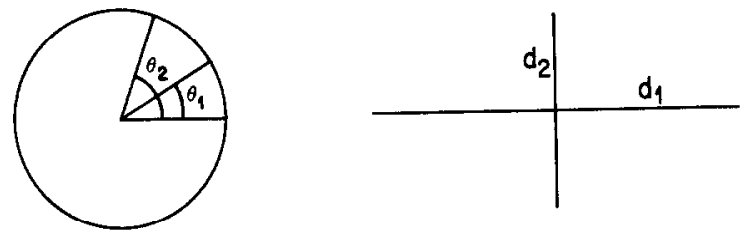

(B)

(c)

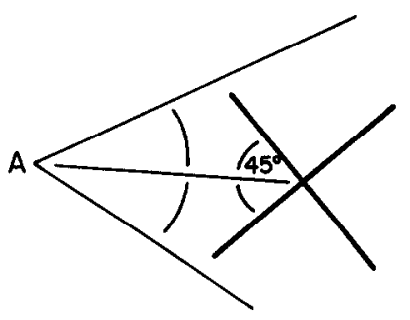

Fig. 10. Invariant angles. A, Angles straddling the axis of compression open; those straddling the axis of extension close. B, Those angles are constant for which $\tan \theta_{1} \tan \theta_{2}=d_{2} / d_{1}$. C, For small changes in shape these are angles bisected by the bisectors of the principal axes.

as the composition of a pure size change, altering nothing but scale, and a pure shape change, leaving area alone. Even though form cannot be decomposed as "shape plus size," form change can be so decomposed, triangle by triangle.

Biorthogonal analysis prescribes a mutual orientation for the two triangles - that for which the principal axes are aligned. We may draw this out, superposing at any pair of homologous points, as in Fig. 8. In the rotation of homologous segments between the triangles, we note the confusion generated by orienting on 

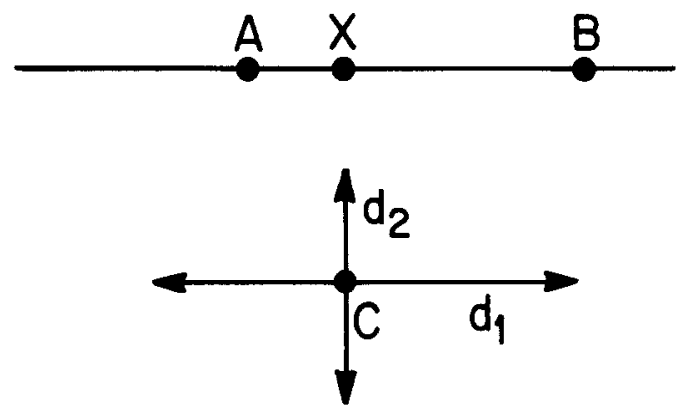

Fig. 11. Invariant division of a segment. The proportion $A X: X B$ is unchanged under deformation.

any single interlandmark segment. All such superpositions are misleading in their depiction of apparent motions of the vertices; starting position is confounded with change. For instance, in the usual anterior cranial base superposition (which correctly suggests homologies in the growing cranial base), the chin "grows" downward and forward mostly because it is downward and forward, as in Fig. 4, $B$. However, there is an alternate way to speak of displacement which is sound. The principal axes through any vertex $\mathrm{V}$ of the triangle will intersect the opposite side somewhere, either between or outside the segment joining the opposite vertices. After a correction for change in size, $\mathrm{V}$ may be described as being displaced straight toward one of these points dividing the edge in fixed fraction and straight away from the other. This description explicitly corrects for dilatations along the edge of reference, the major factor confounding the conventional registration methods.

\section{THE FOUR KINDS OF INVARIANTS}

The simple geometry of this construction leads quickly to four basic classes of invariants which can be identified in any smooth deformation.

\section{Ratio of sides}

Fig. 9 indicates how directions placed symmetrically with respect to the principal axes are dilatated at the same rate by the shape change. Then the ratio of their lengths is unchanged over the transformation. In particular, if either principal axis of a deformation is parallel to the bisector of one of the triangle's vertex angles, then the ratio of the sides which meet at that vertex will be unchanged.

\section{Angle}

Angles with the axis of compression, or straddling it, as in Fig. 10, $A$, must open; those involving or straddling the axis of extension must shrink. Those which span both axes or neither will increase or de-

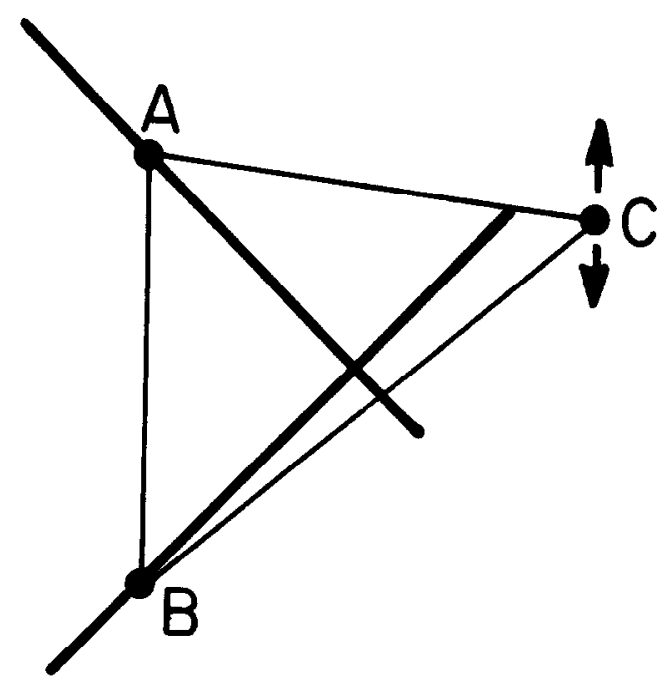

Fig. 12. Invariant ratio of perpendiculars. The two bisecting lines of the principal axes have the same dilatation.

crease as the expanding or shrinking sector dominates. A bit of elementary trigonometry, omitted here, indicales that those angles remain constant for which $\tan \theta_{1}$ $\tan \theta_{2}=\mathrm{d}_{2} / \mathrm{d}_{1}$, ratio of the two dilatations in Fig. 10, $B$. For small changes, where $d_{2} / d_{1}$ is very close to unity, these correspond to angles with $\theta_{1}+\theta_{2}=90^{\circ}$ lines symmetrically placed about the bisectors of the principal axes. Whenever a bisector of the principal cross is aligned with the bisector of one of the triangle's angles (Fig. 10, $C$ ), that angle will remain approximately constant over the transformation.

\section{Proportional division}

If one principal axis is parallel to a side of the original triangle, then the third vertex may be viewed as displaced directly away from the foot of its perpendicular with respect to that side. Hence, whatever the dilatations, the proportion in which the foot of its perpendicular divides that edge is unchanged over the deformation (Fig. 11).

\section{Ratio of perpendiculars}

If each of the principal axes makes an angle of 45 degrees with one side of the starting triangle, then both that side and the altitude to it from the third vertex are dilatated at the same rate (as their directions are symmetric with respect to the axes). Hence, as shown in Fig. 12, the aspect ratio of this triangle, its height divided by its base, will be approximately invariant over the deformation.

\section{Generality of these constructs}

It is not necessary that any of the sides or angle bisectors of the triangle of landmarks lie along the 

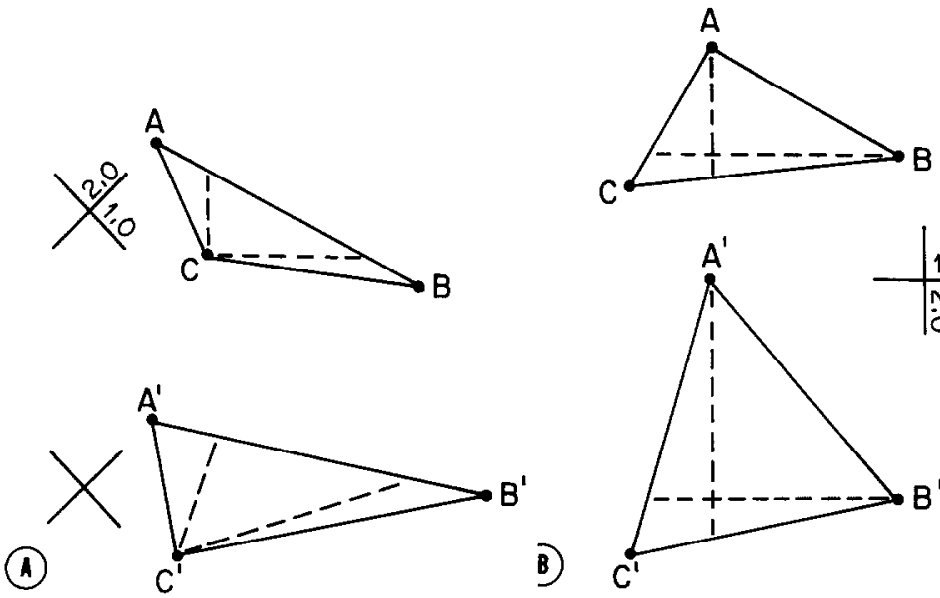

Fig. 13. Invariants involving empirically computed transects. A, Ratio. B, Angle.

Table I. Statistics of population variation $(\mathrm{N}=71)$ in the analyses of Fig. $14^{*}$

\begin{tabular}{|c|c|c|c|c|c|c|}
\hline \multirow[b]{3}{*}{ Triangle, age range } & \multicolumn{4}{|c|}{ Principal dilatations } & \multirow{3}{*}{$\begin{array}{c}\text { Mean } \\
\text { anisotropy }(c)\end{array}$} & \multirow{3}{*}{$\begin{array}{c}\text { Shape fraction } \\
\text { (percent) }(a-b) / c\end{array}$} \\
\hline & \multicolumn{2}{|c|}{ Maximum } & \multicolumn{2}{|c|}{ Minimum } & & \\
\hline & $\operatorname{Mean}(a)$ & S.D. & $\operatorname{Mean}(b)$ & $S . D$. & & \\
\hline \multicolumn{7}{|l|}{ Bas-SER-Men } \\
\hline 6-10 & 2.73 & 0.69 & 1.85 & 0.94 & 1.73 & 51 \\
\hline $10-14$ & 2.73 & 0.79 & 1.30 & 0.86 & 1.82 & 79 \\
\hline \multicolumn{7}{|l|}{ Bas-FMN-Men } \\
\hline $6-10$ & 2.75 & 0.85 & 1.64 & 0.62 & 1.79 & 62 \\
\hline $10-14$ & 2.84 & 0.87 & 1.24 & 0.83 & 1.95 & 82 \\
\hline \multicolumn{7}{|l|}{ Bas-Nas-Men } \\
\hline $6-10$ & 2.75 & 0.81 & 1.69 & 0.46 & 1.66 & 63 \\
\hline $10-14$ & 2.78 & 0.85 & 1.29 & 0.61 & 1.73 & 86 \\
\hline \multicolumn{7}{|l|}{ Sel-FMN-Men } \\
\hline 6-10 & 2.66 & 0.71 & 1.04 & 0.76 & 2.01 & 80 \\
\hline $10-14$ & 2.59 & 0.73 & 0.85 & 1.08 & 2.14 & 81 \\
\hline \multicolumn{7}{|l|}{ Sel-Nas-Men } \\
\hline 6-10 & 2.65 & 0.69 & 1.20 & 0.38 & 1.72 & 84 \\
\hline $10-14$ & 2.53 & 0.71 & 1.03 & 0.46 & 1.70 & 88 \\
\hline \multicolumn{7}{|l|}{ SER-Nas-Men } \\
\hline $6-10$ & 2.71 & 0.64 & 1.52 & 0.64 & 1.71 & 70 \\
\hline $10-14$ & 2.68 & 0.78 & 1.31 & 0.75 & 1.69 & 81 \\
\hline
\end{tabular}

*All columns except the last are in units of percent per year.

principal axes or their bisectors for there to be invariants. We may define homologous segments through any points of the triangles, not only those which happen to be named landmarks. In Fig. 13, $A$, for instance, the lines at 45 degrees to the principal axes are parallel to transects connecting vertex $\mathrm{A}$ to points 0.2 and 0.8 of the distance from vertex $B$ to vertex $C$, segments whose ratio is therefore unchanged by the deformation. In Fig. $13, B$, the principal axes lie along lines from $A$ to a point 0.7 of the way from $B$ to $C$ and from $B$ to a point 0.8 of the way from $A$ to $C$, so the angle between these lines is exactly unchanged at 90 degrees over the deformation. A slowly changing length-ratio corresponds to a rapidly changing angle, and conversely. In Fig. 13, $A$, as the dotted lines are dilatated at the same rate, the angle between them has altered a great deal. In Fig. 13, $B$, because the dashed lines are at an invariant angle of 90 degrees, the proportion between them has altered more than any other shape measure. It thereby becomes a best scalar for discriminating before from after, diagnosing anomalies,${ }^{10}$ characterizing treatment effects, ${ }^{6,8}$ etc.

\section{ANALYSIS OF POPULATIONS}

As explained so far, the biorthogonal method examines the growth of one particular triangle into another. It must be extended to whole populations of shape 

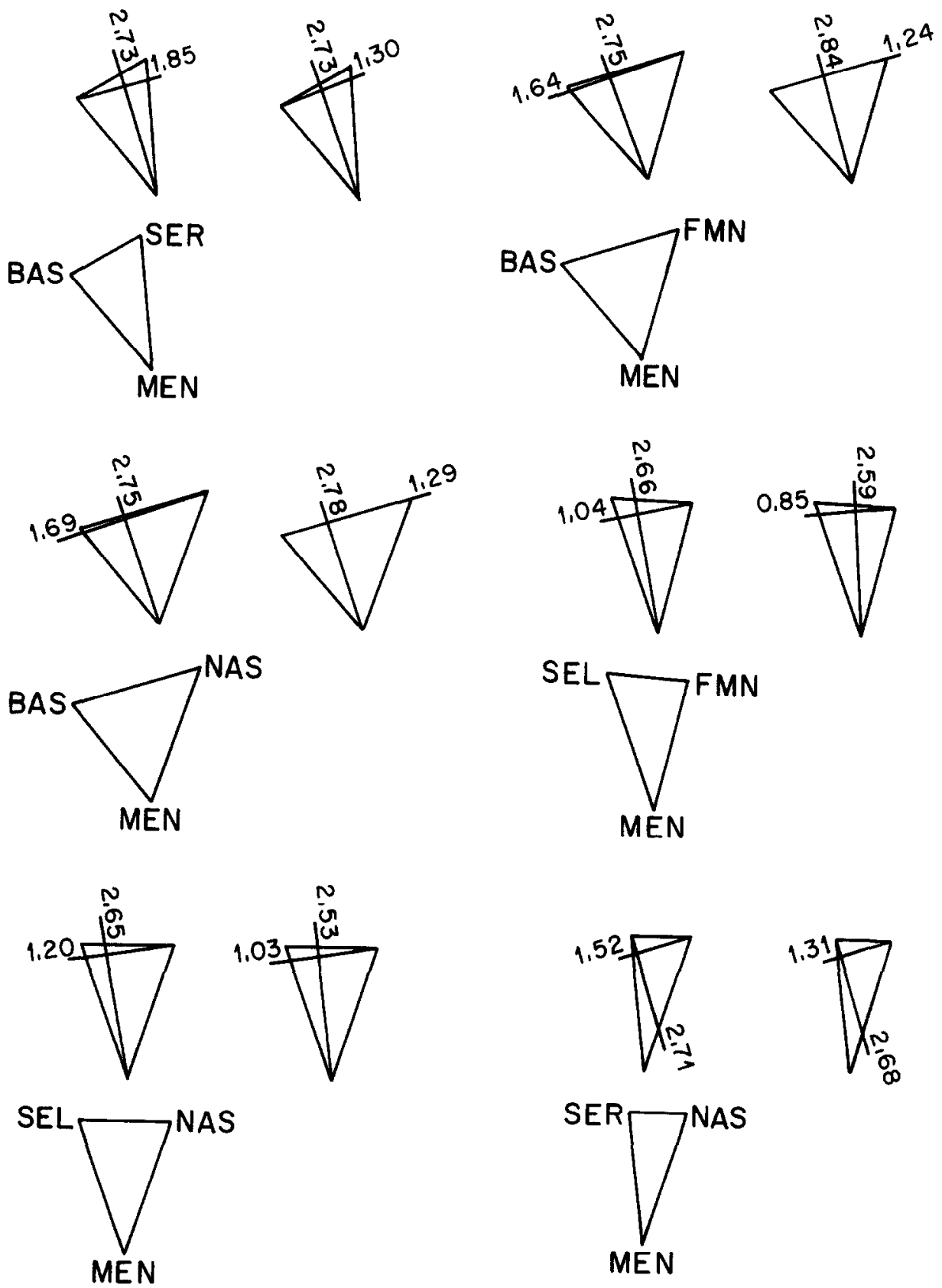
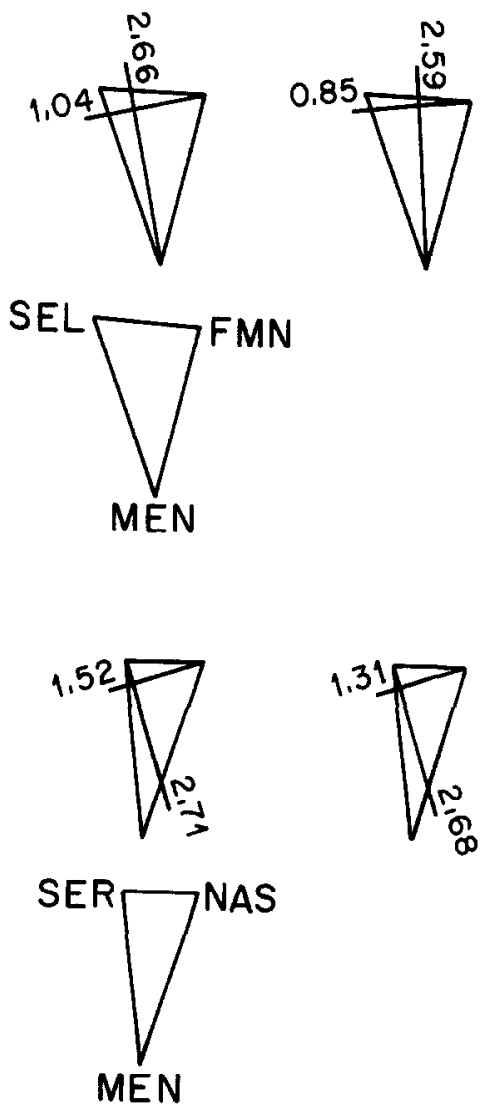

Fig. 14. Six mean analyses of displacement of menton from the cranial base in seventy-one normal Ann Arbor children over two age ranges. For each triangle, the left-hand diagram represents mean growth between ages of 6 and 10 years; the right-hand diagram shows mean growth between 10 and 14 years.

changes. As the computation of averages is routine only for numbers, not geometric objects, we must reduce each principal cross to a collection of parameters. The most effective procedure ${ }^{6}$ records the whole circle of dilatations, after the fashion of Fig. 7, $B$, and averages there.

The linear machinery associated with that diagram let us determine homologies of segments in pairs of forms related by growth. We can use the same uni- form deformation to relate segments in different starting forms, whether related by growth or not: we define homology of direction by equality of the fractions in which segments through a vertex divide the edges opposite. Just as for growth, all dilatations for each edge of the original triangle of landmarks are homologous; for each of the three median lines; and so on. (This is not an even correspondence with respect to angle, but explicitly requires the triangles for its computation.) In 
this manner, we assemble many different sets of dilatations, each set describing the effect of deformation on one single direction throughout the population. Finally, in each homologous family of directions, we average all the observed dilatations to arrive at an average rate of change in length in that direction.

The mean dilatations will approximately describe another ellipse, the "average"' strain. This average will have directions of maximum and minimum dilatation, specified homologously by the fractions in which segments through a vertex divide the edges opposite, that are at approximately 90 degrees to each other in most forms of the series.

If all the crosses for all the shape changes of a population are aligned in the same direction (as determined by our homology convention), the maximum of the mean dilatations would be the mean of the separate maximums, and likewise the minimum. The anisotropy of the mean change (in Table I the difference of columns $a$ and $b$ ) would then be just the mean of the anisotropies of the separate changes being averaged (column $c$ of Table I), and all the invariants of the mean change would devolve on the individuals. If the directions of extreme dilatation for the individual deformations wander far from mutual alignment, then the averages will mix extremal dilatations along principal directions of certain shape changes with middling dilatations along others, attenuating the extremes of those averages and blurring the mean invariants in their application to individuals. The ratio of anisotropy of the means to mean anisotropy is a useful statistic, a sort of "fraction of variance explained by shape change."

\section{EXAMPLES}

What is the best definition of growth direction?

It is convenient to conceive of a "growth direction" at the chin, the general vector of its displacement from the center of the head. This is customarily computed in a sella-nasion superposition. ${ }^{9}$ In any such superposition the apparent displacement of menton confounds changes in the geometry of the cranial base itself - elongations of S-N or drift of nasion upwardwith the phenomenon we are purporting to measure at the chin. There are five landmarks on the cranial base relative to which we might measure displacement of menton: basion (Bas), sella (Sel), spheno-ethmoidal registration (SER), frontomaxillonasal suture (FMN), and nasion (Nas). (For operational definitions of these points, see Riolo and associates. ${ }^{12}$ )

From these five points we may determine ten cranial base segments to which the displacement of menton may be referred. Six of the segments between these points are long enough to serve as stable base lines:
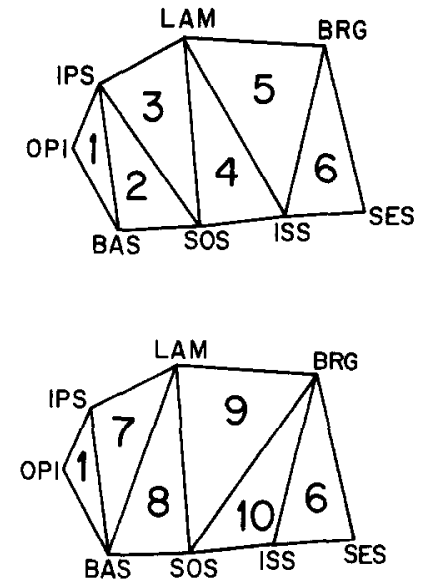

(1)
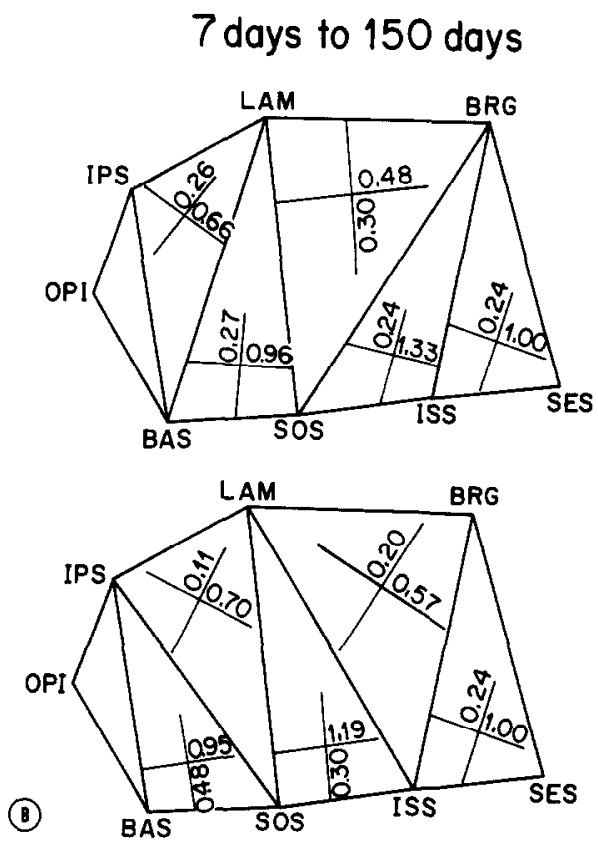

Fig. 15. Growth of twenty-one male rat calvaria between the ages of 7 days and 150 days. A, The landmarks and ten triangles in two triangulations. B, Mean tensor analyses of all ten triangles.

Bas-SER, Bas-FMN, Bas-Nas, Sel-FMN, Sel-Nas, and SER-Nas. The mean analyses for these six configurations, drawn in Fig. 14, pertain to the seventy-one subjects from the University School Study of Moyers and colleagues $^{9}$ who had had films taken in each of the three age ranges 6 to 8,10 to 12 , and 14 to 16 years. Observed changes were computed between the first and second of these films and also between the second and third. In Fig. 14 and in Table I they are reported in annualized form, with the dilatations printed as percentages. For instance, " 2.73 " refers to extension by 2.73 percent per year. For this preliminary study, the sexes are pooled.

All the analyses are consistent with a common 


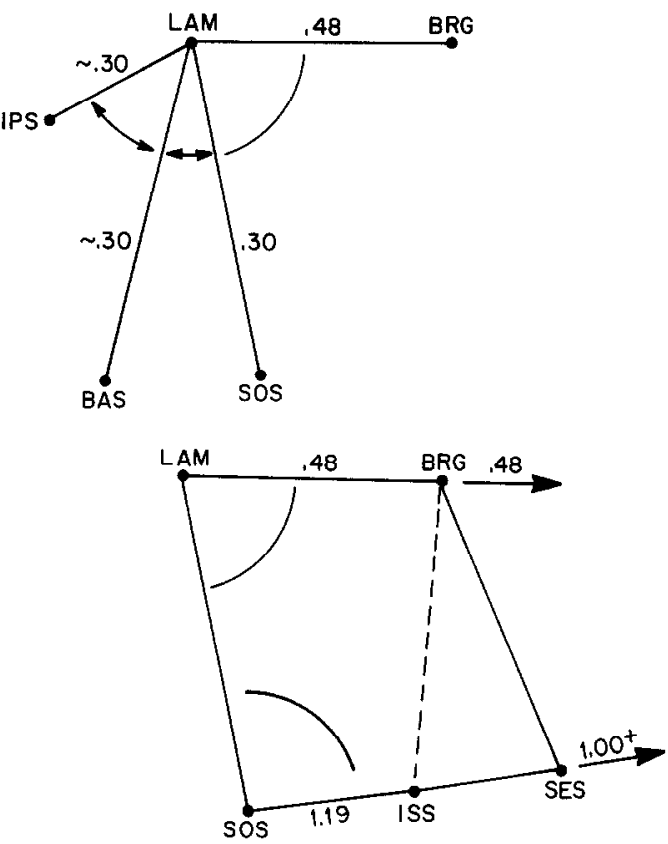

Fig. 16. Abstraction of Fig. 15 into a pattern of two regimes sharing the element lambda-SOS.

principal cross which does not rotate from the first age interval to the second. The direction of largest dilatation is aligned with the "growth axis" that various workers have recognized informally for many years. Menton is displaced away from cranial base along this axis at a rate of 2.5 to 2.8 percent per year. The direction of least dilatation is at 90 degrees to this, closely aligned with Bas-Nas regardless of the cranial base landmarks used in its computation. Small differences in the dilatations from triangle to triangle express the remodeling of the cranial base: the segment basion-sella is itself growing fastest, then SER-Nasion; sella-SER is growing most slowly. Notice how the detection of extremal rates of change of length from menton is tied to the detection of invariant axes "along which" or "away from which" menton is growing.

Table I presents statistics useful in discriminating among the analyses. For each principal dilatation (columns $a$ and $b$ ) there is displayed its standard deviation in the population of 71 , and for each triangle the mean difference (c) between principal dilatations. The ratio $(a-b) / c$ of the difference between the mean principal dilatations to this mean anisotropy is "the fraction of variance explained by shape change." All these help measure the precision with which the exact invariants of the average shape change might be invariant for typical individuals in the population.

The triangle with the least population variance about the common growth analysis is Sel-Nas-Men, with 84 percent of change explained by the systematic shape component in the first time interval and 88 per-
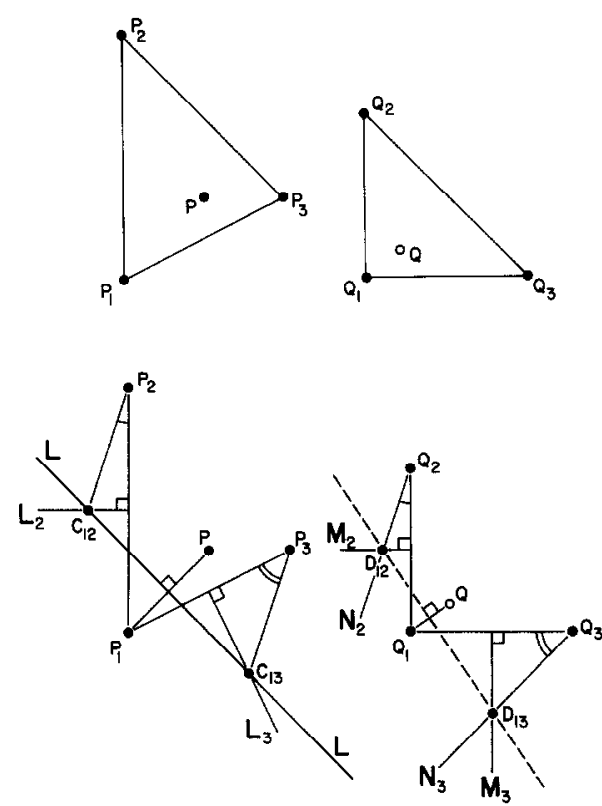

Fig 17. Top: Two triangles and a point $P$. Bottom: The six-step construction of the point $Q$ which, with $P$, forms a pair of isogonal centers.

cent in the second. This triangle also has the least population variance of those principal dilatations themselves. The invariant direction through menton that we seek is best described as passing through a point some 25 percent of the way from sella to nasion. (For the age range 6 to 10 , this fraction is 0.20 ; for 10 to 14 , it is 0.30.) This direction passes rather near to Ricketts' point PT (Fig. 5), the uppermost point of the pterygomaxillary fissure; however, analyses based on that point are noisier than any of those in Fig. 14. Likewise, on average, menton's "growth axis" is perpendicular to the line Bas-FMN or Bas-Nas connecting the extremes of the cranial base, but the precision of representing menton's displacement with respect to that base line is not as high as by Sel-Nas. Basion appears to have a growth direction of its own which, while modest, is nevertheless independent of the changes at menton that we seek to describe and thus contributes error to the description. Growth is more precisely perpendicular to a line joining nasion with a point 10 percent of the way from sella to menton. The single best population invariant would appear to be the proportion in which the foot of the perpendicular from menton divides that segment.

Moyers and Bookstein ${ }^{9}$ explore the stability over time of apparent individual differences in this growth direction.

\section{Growth of the rat skull}

For a second demonstration of the search for growth invariants and corresponding optimal ratios, let us turn to some fully longitudinal data kindly loaned to me by 
Melvin Moss and Henning Vilmann: Cartesian coordinates for eight calvarial landmarks of twenty-one male rats at the ages of $7,14,21,30,40,60,90$, and 150 days postpartum. The landmarks are all intersections of sutures or articulations with the midsagittal plane. Clockwise from lower left in Fig. 15, they are basion (Bas), the lowest point on the foramen magnum; opisthion (Opi), the lowest point on the parietal bone; the intraparietal suture, IPS; lambda (Lam) and bregma ( $\mathrm{Brg})$, intersections of the midsagittal suture with the lambdoidal and coronal sutures; and the centers of the spheno-ethmoidal, intrasphenoidal, and spheno-occipital synchondroses SES, ISS, SOS. These landmarks delimit an octagon which is triangulated in two ways (Fig. 15, A). The growth of these triangles over the whole period of observation, from 7 days to 150 days, is diagrammed in Fig. 15, $B$. (The variation in this summary geometry as a function of age will be reported in a later paper.) In this figure dilatations are fractions, not percentages, of change. For instance, the decimal " 1.19 " in the figure stands for increase by 119 percent, dilatation by the factor 2.19 , over 143 days.

To make sense of this pattern, one might begin by considering the principal dilatations that are easiest to describe, those along segments joining landmarks. One finds principal axes along the cranial base which have extended considerably: Bas-SOS (0.95), SOS-ISS (1.19). Dilatation is least in the direction orthogonal to these. For instance, the increase in the distance LamSOS is a mere 30 percent over the whole 143 days of data.

The three posterior triangles involving lambda (Nos. 7, 8, and 3) bear principal axes directly along appropriate angle bisectors at lambda; their three edges out of lambda must be growing at identical rates (and likewise the two edges through lambda of triangle No. 5). The angles they span at lambda are changing rapidly. A fourth triangle, No. 9, bears principal axes at 45 degrees to the bisector at lambda and thus indicates invariance of the angle (about 90 degrees) there. The changes in these four triangles are summarized in the diagram at the top of Fig. 16.

From this same triangle, No. 9, the one showing the least net shape change of all ten considered, one sees that the point SOS may be said to move almost "straight down" relative to the segment Lam-Brg. Triangle No. 4 corroborates this report, showing lambda to move "straight up" from the sphenoid. The element lambda-SOS preserves its angles to horizontal elements at both end points and thus serves as a useful orientation. The separation of bregma from lambda has increased by an additional fraction of 0.48 over this period, while the separation of ISS from SOS has increased in a parallel direction by a factor of 1.19 . The excess of horizontal growth at the cranial base (119 percent versus 48 percent) is consistent with familiar descriptions of this shape change as orthocephalization. If that process is considered instead as a rotation, the one of our eight landmarks closest to the "center" is apparently bregma. The angle at Bregma in triangle No. 10, for instance, is nearly doubled over the shape change.

Triangle No. 6, the one involving SES, is seen to alter by nearly the same principal cross which describes the change of its neighbor, triangle No. 10. By this means, the angle of the cranial base at ISS is maintained at nearly 180 degrees while the segments SOSISS and SES-ISS grow at relatively large rates.

In light of all these considerations and invariants, the net change of the central calvarial form in the growing rat may be decomposed into two simpler processes: one dealing with changes of angle at lambda together with fairly uniform radial growth away from it, and one dealing with inhomogeneous shear anterior to and perpendicular to the segment Lam-SOS. Fig. 16 shows the resulting analysis. The element Lam-SOS is the best reference line for the study of this calvarial growth geometry. The behavior of triangle No. 1, which includes the foramen magnum, does not seem to relate to the rest of the polygon in any immediately interpretable way.

\section{CONCLUDING REMARKS: ON CEPHALOMETRIC ANALYSIS}

For data in the customary cephalometric formlandmarks located on a cephalogram-there are available many "analyses": specific distances and angles referred to "norms" for diagnosis or compared over time for assessment of growth or treatment. In this essay, which deals with growth, and also in my other essays, ${ }^{7,8}$ which deal with treatment effects, I have attempted to show that description of deformation should come before the measurement of separate forms. The specification of variables of an "analysis," in other words, ought to come only after the assessment of typical shape changes; hence, most published schemes of cephalometric analysis are biometrically premature.

In the analysis of the growth axis, the first of the examples here, we saw clear differences in the reliability of invariants based in alternate sets of landmarks. Between the ages of 6 and 10 , the geometry in terms of which the growth axis is most easily talked about"the perpendicular to basion-nasion through PT" represents its invariance less than half as precisely as the sella-nasion geometry in terms of which that direction can be most accurately determined. For a different scientific purpose, such as treatment assessment, a different geometry of measurement would be indicated. The cervical treatment for Class II, for instance, ${ }^{8}$ tends 
to hold invariant (at 50:50) the proportion in which the perpendicular from menton divides sella-nasion, but drops menton away from that midpoint at an extra 1 percent per year.

In the example of the rat calvarium data, the tensor method leads directly to a collection of invariant ratios and optimal rates characterizing the general growth trend quite tersely. The measures sketched in Fig. 16 constitute a new "analysis" specifically designed to describe growth from 7 days to 150 days, and thereby quite different from the usual lists of measures of the individual calvarium. The measures suited to assessing progress along this mean path would interchange angles and proportions as described above. These analyses change considerably as the time span to which they refer is varied; the comparisons provide a more powerful description of temporal staging of growth than any analysis using a fixed set of variables.

From any set of landmarks indefinitely many cephalometric analyses can be specified wholly arbitrarily. To design an analysis that is not likewise wholly arbitrary, one must select measures in light of the discrimination or trend that the measures are intended to report. Analyses should be of form-change, not of form; the measure(s) selected ought to be most sensitive to some trend or contrast or else not express it at all. No other variables need claim our morphometric attention. Changing most or changing not at all: these are just the findings thrust at us by the mean biorthogonal method.

I acknowledge the support of National Institutes of Health Grants DE-05410 to me, HD-14371 to Melvin Moss, and DE- 03610 to $R$. F. Moyers in the course of the research reported here.

\section{Appendix}

For two triangles of landmarks $\mathrm{P}_{1} \mathrm{P}_{2} \mathrm{P}_{3}, \mathrm{Q}_{1} \mathrm{Q}_{2} \mathrm{Q}_{3}$, and any point $P$ in the plane of the first triangle (Fig. 17, top), here is how to construct a point $Q$ such that $\angle Q_{1} Q_{2}=$ $<\mathrm{P}_{1} \mathrm{PP}_{2},<\mathrm{Q}_{2} \mathrm{QQ}_{3}=<\mathrm{P}_{2} \mathrm{PP}_{3},<\mathrm{Q}_{3} \mathrm{QQ}_{1}=<\mathrm{P}_{3} \mathrm{PP}_{1}$, so that $\mathrm{P}$ and $\mathrm{Q}$ will form a pair of isogonal centers:

Construct the perpendicular bisector $L_{1}$ of line $P_{1} P$ (Fig. 17, bottom). Intersect $\mathrm{L}_{1}$ with the perpendicular bisectors $\mathrm{L}_{2}, \mathrm{~L}_{3}$ of $\mathrm{PP}_{2}$ and $\mathrm{PP}_{3}$ to get points $\mathrm{C}_{12}, \mathrm{C}_{13}$. Erect the perpendicular bisectors $\mathrm{M}_{2}, \mathrm{M}_{3}$ of segments $\mathrm{Q}_{1} \mathrm{Q}_{2}, \mathrm{Q}_{1} \mathrm{Q}_{3}$. Construct the line $\mathrm{N}_{2}$ through $\mathrm{Q}_{2}$ making the same angle with $Q_{1} Q_{2}$ as line $L_{2}$ makes with $P_{1} P_{2}$, and let this line $N_{2}$ intersect $M_{2}$ at the point $D_{12}$. Likewise, construct the line $\mathrm{N}_{3}$ through $\mathrm{Q}_{3}$, making the same angle with $Q_{1} Q_{3}$ as line $L_{3}$ makes with $P_{1} P_{3}$, and let this line $N_{3}$ intersect $M_{3}$ at the point $D_{13}$.

The point $\mathrm{Q}$ such that $\mathrm{P}$ and $\mathrm{Q}$ form a pair of isogonal centers is the reflection of $Q_{1}$ in the line $D_{12} D_{13}$.
Proof. The points $\mathrm{X}$ with $<\mathrm{P}_{1} \mathrm{XP}_{2}=<\mathrm{P}_{1} \mathrm{PP}_{2}$ lie on the circle through $\mathrm{P}_{1}, \mathrm{P}, \mathrm{P}_{2}$. This circle is centered at $\mathrm{C}_{12}$. Since the triangle $\mathrm{D}_{12} \mathrm{Q}_{1} \mathrm{Q}_{2}$ is similar to the triangle $\mathrm{C}_{12} \mathrm{P}_{1} \mathrm{P}_{2}$, by construction, for all points $\mathrm{Y}$ on the circle through $\mathrm{Q}_{1}$ and $\mathrm{Q}_{2}$ centered at $\mathrm{D}_{12},<\mathrm{Q}_{1} \mathrm{YQ}_{2}=$ $<\mathrm{P}_{1} \mathrm{PP}_{2}$. Similarly, for points $\mathrm{Y}$ on the circle centered at $\mathrm{D}_{13}$ through $\mathrm{Q}_{1}$ and $\mathrm{Q}_{3},<\mathrm{Q}_{1} \mathrm{YQ}_{3}=<\mathrm{P}_{1} \mathrm{PP}_{3}$.

We seek the point $Q$ on the intersection of these two circles, but they intersect once at $Q_{1}$, we know. The other intersection $Q$ must then be the reflection of $Q_{1}$ in the line $\mathrm{D}_{12} \mathrm{D}_{13}$ connecting the centers of the circles.

\section{REFERENCES}

1. Ricketts, R. M.: Mechanisms of mandibular growth: A series of inquiries on the growth of the mandible. In McNamara, J. A., Jr. (editor): Determinants of mandibular form and growth, Monograph No. 4, Craniofacial Growth Series, Ann Arbor, 1975, Center for Human Growth and Development, University of Michigan, pp. 77-100.

2. Moss, M. L., Skalak, R., Moss-Salentijn, L., Dasgupta, G., Vilmann, H., and Mehta, P.: The allometric center: The biological basis of an analytical model of growth, Proc. Finn. Dent. Soc. 77: 119-128, 1981 .

3. Todd, J. T., and Mark, L. S.: Issues related to the prediction of craniofacial growth, AM. J. ORTHOD. 79: 63-80, 1981.

4. Bookstein, Fred L.: Superposition: Analytic geometry in the study of growth. In Antonelli, P. (editor): Proceedings of the Conference on Diffusion and Geometry in Population and Developmental Biology, Edmonton, Alta., 1983, University of Alberta Press. (In press.)

5. Moss, M. L., Skalak, R., Shinozuka, M., Patel, H., MossSalentijn, L., Vilmann, H., and Mehta, P.: The testing of an allometric center model of craniofacial growth. In Burstone, C. F., and Nanda, R. S. (editors): Clinical and bioengineering aspects of dentofacial orthopedics. (In press.)

6. Bookstein, Fred L.: Foundations of morphometrics, Ann. Rev. Ecol. Systematics 13: 451-470, 1982.

7. Bookstein, Fred L.: On the cephalometrics of skeletal change, AM. J. ORTHOD. 82: 177-198, 1982.

8. Bookstein, Fred L.: Measuring treatment effects on craniofacial growth. In Carlson, D. S. (editor): Clinical alteration of the growing face, Craniofacial Growth Series, Ann Arbor, 1983, Center for Human Growth, University of Michigan, pp. 65-80.

9. Moyers, R. E., and Bookstein, Fred L.: Do horizontal growers grow horizontally? Swed. Orthod. J. (In press, 1982.)

10. Grayson, B., Bookstein, F., Kolber, A., and McCarthy, J.: Measuring craniofacial deformity in the single case. (In preparation.)

11. Bookstein, Fred L.: The measurement of biological shape and shape change, Lecture notes in biomathematics, vol. 24, Berlin, 1978, Springer-Verlag.

12. Riolo, M. L., Moyers, R. E., McNamara, J. A., and Hunter, W. S.: An atlas of craniofacial growth, Monograph No. 2, Craniofacial Growth Series, Ann Arbor, 1974, Center for Human Growth and Development, University of Michigan.

Reprint requests to:

Dr. Fred Bookstein

Center for Human Growth and Development

University of Michigan

300 North Ingalls Bldg.

Ann Arbor, Mich. 48109 